\title{
ANÁLISE ESPACIAL DAS AGROINDÚSTRIAS DA REGIÃO SUL: UM ESTUDO A NÍVEL MUNICIPAL PARA O ANO 2010
}

\author{
Ana Paula Buhse ${ }^{1}$ \\ Tatiane Pelegrini ${ }^{2}$ \\ Adelar Fochezatto ${ }^{3}$
}

\begin{abstract}
Resumo: Este trabalho teve como objetivo principal verificar os padrões geográficos das indústrias do agronegócio dispostos nos municípios da região sul do Brasil para o ano de 2010, a fim de apurar se ocorre o fenômeno de concentração/aglomeração agroindustrial em torno das cidades centrais. Como base metodológica utilizou-se a Análise Exploratória de Dados Espaciais (AEDE) e econometria espacial. Os resultados demonstraram a existência de autocorrelação espacial positiva entre os municípios da região sul. Ao constatar dependência espacial, os modelos econométricos considerados mais adequados foram Modelos de Lag espacial (SAR) e o Modelo de Erro Espacial (SEM), no qual indicaram sinal positivo e significativo para as variáveis VAB agropecuário e da indústria; renda per capita; população total e rural. Os resultados indicam que as agroindústrias estão se concentrando nos principais centros de cada estado em estudo, ou seja, as empresas estão próximas de locais com maior população, o mercado consumidor.
\end{abstract}

Palavras-chave: Agroindústrias; Região sul; Análise Exploratória de Dados Espaciais; Econometria Espacial

\section{ANALYSIS OF SPACE SOUTH REGION AGRIBUSINESSES: A STUDY THE MUNICIPAL GOVERNMENT FOR THE YEAR 2010}

\begin{abstract}
This work aimed to check the geographic patterns of agribusiness industries distributed on municipalities in the south of the Brazil for the year 2010 in order to determine whether there is the phenomenon of concentration/agroindustrial crowding around the central cities. The methodology used is Exploratory Analysis of Spatial Data (ESDA) and spatial econometric. The results showed the existence of positive spatial autocorrelation between the municipalities of the southern region. With spatial dependence, the more appropriate econometric models were the spatial lag models (SAR) and Model Space error (SEM), which indicated positive sign and significant value for agricultural variables and industry; income per capita; and total rural population. In the results, the agricultural industries are focusing in the main centers of each state under study, that is, companies are close to areas with larger population, the consumer market.
\end{abstract}

Keywords: Agribusiness; South region; Exploratory Spatial Data Analysis; Spatial Econometrics

\footnotetext{
${ }^{1}$ Doutoranda do Programa de Pós Graduação de Economia na PUCRS. Email: anapaulabuhse@ hotmail.com

${ }^{2}$ Doutoranda do Programa de Pós Graduação de Economia na PUCRS. Emal: tatikpelegrini@gmail.com

${ }^{3}$ Professor titular da Pontifícia Universidade Católica do Rio Grande do Sul - PUCRS. Email: adelar@pucrs.br
} 


\section{ANÁLISIS ESPACIAL DE LAS AGROINDUSTRIAS DE LA REGIÓN SUR: UN ESTUDIO A NIVEL MUNICIPAL PARA EL AÑO 2010}

Resumen: Este trabajo tuvo como objetivo principal verificar los patrones geográficos de las industrias del agronegocio dispuestos en los municipios de la región sur del país para el año 2010, a fin de averiguar si ocurre el fenómeno de concentración/aglomeración agroindustrial en torno a las ciudades centrales. Como base metodológica se utilizó el Análisis Exploratorio de Datos Espaciales (AEDE) y econometría espacial. Los resultados demostraron la existencia de autocorrelación espacial positiva entre los municipios de la región sur. En el caso de la dependencia espacial, los modelos econométricos considerados más adecuados fueron Modelos de Lag espacio (SAR) y el Modelo de Error Espacial (SEM), en el cual indicaron señal positiva y significativa para las variables VAB agropecuario y de la industria; renta per cápita; población total y rural. Los resultados indican que las agroindustrias se concentran en los principales centros de cada estado en estudio, o sea, las empresas están cerca de locales con mayor población, el mercado consumidor.

Palabras clave: Agroindustrias; Región sur; Análisis Exploratorio de Datos Espaciales; Econometría Espacial

\section{INTRODUÇÃO}

O agronegócio pode ser interpretado como um conjunto de atividades derivadas e dependentes da produção agropecuária, que representa uma cadeia produtiva que envolve desde a fabricação de insumos até o seu consumo final (GASQUES et. al, 2004). De acordo com Canuto (2004), o agronegócio tem sido apresentado como modelo de modernização, onde seu desenvolvimento pode ser expresso pela aplicação de tecnologia no campo, pelos ganhos de produtividade e incrementos de receita econômica.

Tido como um dos principais e mais dinâmicos setores de atividade econômica no Brasil, o agronegócio apresentou um crescimento acumulado de 4,48\% no ano de 2016, enquanto que o PIB brasileiro registrou um recuo de 3,6\% no mesmo ano. Este crescimento foi possível graças a valorização acumulada dos preços, especialmente para os setores primários (CONFEDERAÇÃO DA AGRICULTURA E PECUÁRIA NO BRASIL, 2017).

A produção agropecuária registrou um valor bruto de produção de $\mathrm{R} \$ 527,9$ bilhões em 2016, sendo que a região sul liderou o valor com R $\$ 155,78$ bilhões, o que corresponde a $29,5 \%$ do total, seguida pela região sudeste $(27,5 \%)$ e centro-oeste $(29,5 \%)$. A cadeia agropecuária também é essencial na economia brasileira pela promoção de divisas. Ainda em 2016, as exportações do complexo representaram 45,9\% do total das exportações, gerando um saldo de US\$ 71,3 bilhões para a Balança Comercial (BRASIL, 2017).

Em função da grande importância do agronegócio para o atual contexto socioeconômico brasileiro, este artigo tem como objetivo principal verificar os padrões 
geográficos de aglomeração espacial das agroindústrias dos municípios da região sul, maior produtora brasileira, para o ano de 2010. A análise é feita por meio do número de estabelecimentos dispostos em todos os municípios da região, a fim de apurar se ocorre um fenômeno de concentração/aglomeração agroindustrial em torno de cidades centrais.

A base teórica deste estudo é fundamentada por Weber (1929), que determina os principais pressupostos da teoria da localização industrial, ferramenta significativa para estabelecer a instalação de indústrias. De acordo com o autor, o principal objetivo é a minimização dos custos, considerando três fatores fundamentais que influenciam na decisão locacional: os custos de transporte, os custos de mão-de-obra e as forças de aglomeração e desaglomeração.

Entre os trabalhos que identificam a localização espacial da produção agropecuária e das agroindústrias, podem-se elencar: Haddad (1999); Lopes e Caixeta Filho (2000); Ostroski e Medeiros (2004); Souza e Perobelli (2007); Carmo, Soares e Lopes (2011); Kusbick e Tartas (2015). O estudo de Haddad (1999) conceitua e analisa a formação de clusters voltados ao agronegócio em diversas regiões do Brasil. A pesquisa procurou identificar as áreas geográficas relevantes para cada um dos clusters selecionados, por meio de dados de indicadores de desempenho setorial, infra-estrutura física de apoio e serviços de suporte empresarial, além de avaliar aspectos inerentes ao desenvolvimento social local e aos impactos ambientais.

Via modelo de programação mista, Lopes e Caixeta Filho (2000) analisaram fatores locacionais para a distribuição mais eficiente de granjas suinícolas no estado de Goiás. Por meio da construção de diferentes cenários relacionados ao consumo per capita de carne suína, os autores verificaram uma tendência a implantação de granjas de grande porte localizadas nas proximidades dos abatedouros, gerando economias de escala na região e reduzindo os custos de transporte de matéria-prima.

Por meio de uma análise bibliográfica acerca das definições e características de agriclusters, Ostroski e Medeiros (2004) verificaram se existem, por meio de entrevistas com representantes da cadeia no município de Toledo-PR, fatores que caracterizam o município como cluster agroindustrial suinícola. A análise exploratória de dados espaciais realizado por Souza e Perobelli (2007) compreendeu a distribuição espacial da produção de café para as microrregiões brasileiras no período de 1991 a 2003. A estrutura espacial de produção do café demonstrou-se espacialmente concentrada, com a presença de quocientes locacionais elevados 
de produção nos estados de Minas Gerais, Espírito Santo e em microrregiões do estado de Rondônia.

Carmo, Soares e Lopes (2011) utilizaram a análise espacial como ferramenta na tomada de decisões e intervenções no espaço. Por meio de um Sistema de Informações Geográficas foi possível determinar a localização ótima de um empreendimento, sendo explorado no trabalho o caso de uma agroindústria avícola localizada no Distrito Federal. Os resultados indicaram que os abatedouros deveriam estar localizados próximos aos centros de distribuição e aos clientes sujeitos às restrições locais, uma vez que o objetivo principal é a minimização dos custos de localização, transporte e produção.

Por fim, a teoria de localização industrial weberiana foi considerada na exploração dos principais fatores de implantação da agroindústria no oeste catarinense realizada por Kusbick e Tartas (2015). Na região foi encontrada elevada concentração de agroindústrias da cadeia suinícola e avícola, uma vez que apresentava uma tradicional produção e comercialização de animais, bem como capital, apoio governamental, disponibilidade de mão-de-obra e matériaprima, além de empreendedorismo por parte dos industriais.

Ao considerar os trabalhos supracitados é possível verificar uma predominância na utilização de técnicas de análises que buscam identificar padrões geográficos de aglomeração de agroindústrias e produção agropecuária. Os estudos sobre o setor agroindustrial têm tido destaque, uma vez que o mesmo está associado a economias de aglomeração e desenvolvimento econômico nas regiões onde está presente, no entanto, os mesmos apresentam heterogeneidade na análise dos setores e regiões, especialmente quanto aos fatores sociais, culturais, de infra-estrutura e de gestão organizacional (GUIMARÃES, 2006).

Ao diagnosticar a importância do agronegócio no Brasil, este trabalho dedica-se a verificar o padrão de aglomeração espacial das agroindústrias e se esse padrão apresenta uma correlação significativa com a concentração populacional nos municípios brasileiros situados na região sul. Para atender a este propósito, o artigo está dividido em cinco seções, sendo a primeira esta introdução e a segunda tratando do referencial teórico acerca da definição e relevância da teoria da localização industrial. A terceira parte do trabalho engloba a metodologia empregada a fim de se analisar os resultados que são expressos na quarta seção. Finalmente, na quinta e última parte discutem-se as considerações finais desta pesquisa. 


\section{REFERENCIAL TEÓRICO}

A literatura econômica admite que ocorre tendência à concentração espacial da indústria, dada pelas economias de aglomeração. As teorias da localização, nesse contexto, definem as forças concentradoras das atividades econômicas e outras que agem em sentido contrário, as forças de dispersão. Originadas pelos trabalhos de Weber (1929) e Lösch (1954), as teorias da localização possuem uma hipótese em comum, que as empresas escolhem sua localização baseadas na possibilidade de maior lucro esperado.

A teoria clássica da localização é uma ferramenta desenvolvida para estabelecer o lugar ideal para a instalação de indústrias baseada em fatores gerais, específicos ou motivacionais e permitir o conhecimento locacional de determinado território. Este arcabouço teórico visa orientar a política de desenvolvimento local e oferecer aos investidores bases importantes para o estabelecimento de atividades econômicas (HADDAD, 1989).

A teoria weberiana apresenta uma teoria geral para a localização de uma firma individualmente, sendo que três fatores locacionais influenciam a escolha do local onde serão efetivados os empreendimentos: os custos de transporte, os custos associados a mão-de-obra e as vantagens de aglomeração (WEBER, 1929). A localização ideal, com menores custos de transporte, considera um caso onde existem duas matérias-primas necessárias e situadas em dois pontos diferentes, com um único mercado consumidor localizado em um terceiro ponto, o chamado "triângulo locacional".

Para Weber os determinantes para a localização da empresa são os custos de transportes relacionados à matéria-prima e ao produto final, a localização dos mercados consumidores, das fontes de matéria-prima e de mão-de-obra são dados. O autor considera que "os custos de transportes constituem a única influência sobre a localização" (RICHARDSON, 1981, p. 57), determinando a localização ótima no ponto onde se minimizam os custos de transporte.

Outro autor essencial na teoria da localização industrial é Lösch (1954), que aborda a localização ótima individual das firmas, que devem priorizar a maximização dos lucros. De acordo com Azzoni (1982), a grande contribuição de Lösh foi a análise de um sistema de cidades e a distribuição de atividades no espaço em forma de centros, que apresentam diferentes bens, economias de escala e custos de transporte, compondo uma rede complexa.

O uso da teoria locacional se justifica neste trabalho em função das peculiaridades que enfrentam as empresas ligadas ao agronegócio em relação ao custo de transporte, matériaprima e mão-de-obra. De acordo com Torezani, Andreotti e Campos (2013) o alto volume de 
matérias-primas, além da perecibilidade do produto final torna imprescindível a rapidez e o baixo custo nos transportes e na logística de mercado corroborando a importância desta abordagem para o setor.

Outro ponto que deve ser considerado neste mercado e que valida os estudos que envolvem a localização industrial é a competitividade internacional do setor agropecuário brasileiro, que se destaca pelo potencial exportador e que exige o estudo de diferentes modos de transporte (LOPES e CAIXETA FILHO, 2006). Dessa forma, a localização ótima das agroindústrias é regida por inúmeros fatores e costuma se concentrar em determinados locais que expressam vantagens produtivas e comerciais. A próxima seção determina os aspectos metodológicos utilizados a fim de contemplar a localização das agroindústrias da região sul.

\section{METODOLOGIA}

O artigo utiliza como base metodológica para analisar os padrões geográficos das agroindústrias a Análise Exploratória de Dados Espaciais (AEDE) e Econometria Espacial, expressas nesta seção. Ao final, são apresentadas a descrição das variáveis e a fonte de dados.

\section{Análise Exploratória de Dados Espaciais}

Análise Exploratória de Dados Espaciais está baseada nos aspectos espaciais da base de dados, tratando de forma direta das questões como dependência espacial (e.g associação espacial) e heterogeneidade espacial (PEROBELLI et al.; 2007). A análise torna-se fundamental ao estudo da econometria espacial, uma vez que, lida com as complicações causadas pela interação espacial (autocorrelação espacial) e pela estrutura espacial (heterogeneidade espacial) em modelos de regressão para dados na forma de cross-section e painel de dados (ALMEIDA; 2004).

Com a AEDE é possível analisar medidas de autocorrelação espacial global e local, investigando a influência dos efeitos espaciais por intermédio de métodos quantitativos (ROCHA; PARRÉ, 2009). 


\subsubsection{Autocorrelação Espacial Global}

A presença de autocorrelação espacial é medida usualmente através de estatísticas globais. O índice global Moran's I é a estatística mais difundida e mede a correlação a partir do produto dos desvios em relação à média. Esta estatística fornece a indicação formal do grau de associação linear (espacial) entre os vetores de $Z_{t}$ no tempo $t$ e a média ponderada dos valores da vizinhança, ou lags espaciais $\left(W Z_{t}\right)$ (ANSELIN; 1992).

Conforme Anselin (1992) é especificado da seguinte forma:

$$
\mathrm{I}=\left(\frac{\mathrm{n}}{\Sigma_{\mathrm{i}} \Sigma_{\mathrm{j}} \mathrm{w}_{\mathrm{ij}}}\right) \times\left(\frac{\Sigma_{\mathrm{i}} \Sigma_{\mathrm{j}} \mathrm{w}_{\mathrm{ij}}\left(\mathrm{y}_{\mathrm{i}}-\mathrm{u}\right)\left(\mathrm{y}_{\mathrm{i}}-\mathrm{u}\right)}{\Sigma_{\mathrm{i}}\left(\mathrm{y}_{\mathrm{i}}-\mathrm{u}\right)^{2}}\right)
$$

Onde: $n$ é o número de observações; $W_{i j}$ é o elemento na matriz peso espacial correspondente a unidades espaciais $i$ e $j ; y_{i} e y_{j}$ são observações para os respectivos locais; $\mu$ representa a média de $y$.

O índice varia entre -1 a 1 , sendo que taxas próximas de zero indicam inexistência de autocorrelação espacial significativa; se o valor for positivo (negativo), a autocorrelação será positiva (negativa). Porém, a estatística Moran's I é uma medida global, não sendo possível observar a estrutura de correlação espacial em nível regional, com isso é indispensável a utilização de indicador de autocorrelação espacial local (PEROBELLI et al.; 2007).

\section{Autocorrelação Espacial Local}

A estatística global Moran's I pode esconder padrões locais de autocorrelação espacial, sendo possível ocorrer três situações distintas, são elas: i) envolve a indicação de um I de Moran global insignificante, porém, pode haver indicações de autocorrelação espacial local insignificante, positiva ou negativa; ii) implica uma indicação positiva do I de Moran global, que oculta autocorrelação espacial local negativa e insignificante; e iii) denota que a evidência de uma autocorrelação espacial global negativa e pode acomodar indícios de autocorrelação espacial local positiva para certos grupos dos dados (PEROBELLI et al.; 2007).

É importante avaliar o padrão local da autocorrelação espacial a fim de se obter um maior detalhamento. Para observar a existência de clusters espaciais locais de valores altos ou baixos e quais as regiões que mais contribuem para a existência de autocorrelação espacial, devem-se implementar as medidas de autocorrelação espacial local, tais como: diagrama de 
dispersão de Moran (Moran Scatterplot) e LISA (Indicadores Locais de Associação Espacial) (PEROBELLI et al.; 2007).

\section{Diagramas de Dispersão de Moran}

O diagrama de Moran identifica a tendência geral de associação por meio da correlação linear entre $W_{z}$, que são os valores da variável de interesse defasados espacialmente, e $z$, que são os valores observados em cada unidade espacial (ANSELIN; 1995).

O digrama é divido em quatro quadrantes, os quais correspondem padrões de associação local espacial entre as regiões e seus vizinhos (ANSELIN, 1995). O primeiro quadrante (localizado na parte superior direita) é classificado como alto-alto (AA) (high-high - HH), ou seja, mostra as regiões que apresentam altos valores para a variável em análise cercada por regiões que também apresentam valores acima da média para a variável em análise. O segundo quadrante, baixo-alto (BA) (low-high - LH), (localizado na parte superior esquerda) mostra as regiões com valores baixos cercados por vizinhos que apresentam valores altos (PEROBELLI et al.; 2007).

O terceiro quadrante, classificado como baixo-baixo (BB) (low-low - LL), (localizado no canto inferior esquerdo) é constituído pelas regiões com valores baixos para as variáveis em análise cercados por vizinhos que também apresentam baixos valores, classificado como baixo-baixo. O quarto quadrante, alto-baixo (AB) (high-low - HL), (localizado no canto inferior direito) é formado pelas regiões com altos valores para as variáveis em análise cercados por regiões com baixos valores (PEROBELLI et al.; 2007).

As regiões que apresentam padrões AA e BB possuem autocorrelação espacial positiva e formam clusters de valores similares. Já as que apresentam padrões BA e AB, apresentam autocorrelação espacial negativa, ou seja, formam clusters com valores diferentes (SOUZA; PEROBELLI; 2007).

Indicadores locais de associação espacial (LISA)

O Indicador Local de Autocorrelação Espacial Local é utilizado para mostrar que ocorrem agrupamentos em função de uma vizinhança preestabelecida. Porém, é necessário atender dois objetivos específicos; i) permitir a identificação de padrões de associação 
espacial significativos; ii) a soma dos indicadores de LISA para todas as observações deve ser proporcional ao índice global de associação espacial (ANSELIN; 1995).

A estatística LISA, baseada no I de Moran local pode ser especificada da seguinte forma (LE GALLO; ERTUR; 2003):

$$
\begin{gathered}
I_{i, t}=\frac{\left(x_{i, t}-u_{t}\right)}{m_{0}} \sum_{j} w_{i j}\left(x_{j, t}-u_{t}\right) \\
\operatorname{Com} m_{0}=\frac{\left(x_{i, t}-u_{t}\right)^{2}}{n}
\end{gathered}
$$

Onde: $x_{i t}$, é a observação de uma variável de interesse na região $i$ para o ano $t$; $u_{t}$ é a média das observações entre as regiões no ano $t$ para a qual o somatório em relação a $j$ é tal que somente os valores vizinhos de $j$ são incluídos.

Valores positivos de $I_{i, t}$ significam que existem clusters espaciais com valores similares (alto ou baixo); enquanto, valores negativos indicam que existem clusters espaciais com valores diferentes entre as regiões e seus vizinhos (PEROBELLI et al.; 2007).

Determinação da matriz de pesos espaciais (W)

A matriz de pesos é a forma de expressar a estrutura espacial dos dados e é o ponto inicial para qualquer teste estatístico ou modelo (PEROBELLI et al.; 2007). No trabalho a matriz de peso espacial $\mathrm{W}$ está baseada na ideia dos $k$ vizinhos mais próximos. A escolha da matriz de pesos é importante em uma análise AEDE, pois todos os passos subsequentes são dependentes desta seleção.

Conforme Perobelli et al. (2007), a forma da matriz de pesos espaciais é a seguinte:

$$
\begin{aligned}
& w_{i j}(k)=0 \text { se } i=j \\
& w_{i j}(k)=1 \text { se } d_{i j} \leq D_{i}(k) \text { e } w_{i j}(k) / \sum_{j} w_{i j}(k) \text { para } k=1,2, \ldots, n \\
& w_{i j}(k)=0 \text { se } d_{i j}>D_{i}(k)
\end{aligned}
$$

Onde: $d_{i j}$ é a distância, medida pelo grande círculo, entre os centros das regiões $i$ e $j ; D_{i}(\mathrm{k})$, valor crítico que define o valor de corte, ou seja a distância máxima para considerar regiões vizinhas à região $i$. 
A escolha de um número fixo de vizinhos mais próximos ao invés do uso de uma matriz simples de continuidade é melhor, em função de evitar alguns problemas metodológicos que podem ocorrer quando há variações no número de vizinhos (LE GALLO; ERTUR; 2003).

\section{Modelo econométrico}

No caso de regressão com dados espaciais, ocorrendo autocorrelação espacial, o modelo gerado deve incorporar a estrutura espacial, já que a dependência entre as observações afeta a capacidade de explicação do modelo (CÂMARA et al.; 2002).

Existem diversas maneiras para introduzir os efeitos espaciais em modelos de regressão, sendo a mais simples denominada modelo com efeitos espaciais globais, que busca capturar a estrutura de correlação espacial em apenas um parâmetro e adicioná-lo no modelo de regressão clássico (PRADO et al.; 2010).

A primeira delas é pelo Modelo de Lag Espacial (SAR), que atribui à variável resposta Y a autocorrelação espacial ignorada. O modelo é expresso da seguinte forma:

$$
\mathrm{Y}=\mathrm{X} \beta+\mathrm{pWY}+\varepsilon
$$

Onde: W é a matriz de proximidade espacial; WY expressa a dependência espacial em Y; e $\rho$ é o coeficiente espacial autoregressivo.

A segunda alternativa, segundo Prado et al. (2010), é pelo Modelo do Erro Espacial (SEM), que considera os efeitos espaciais como um ruído, isto é, como um fator a ser removido, sendo expresso da seguinte forma:

$$
\begin{aligned}
& Y=X \beta+\varepsilon, \\
& \varepsilon=\lambda W_{\mathrm{s}}+\xi
\end{aligned}
$$

Onde: $W_{\varepsilon}$ é a componente do erro com efeitos espaciais; $\lambda$ é o coeficiente autoregressivo; e $\xi$ é a componente do erro com variância constante e não correlacionada.

\section{Descrição das variáveis e fonte de dados}

O modelo em estudo tem como variável endógena os estabelecimentos agroindustriais classificados, de acordo com o Instituto Brasileiro de Geografia e Estatística (IBGE), em 
trinta e um setores ${ }^{4}$. Como variáveis exógenas o Valor Adicionado Bruto, a preços correntes, da Indústria (Vab_ind); Valor Adicionado Bruto, a preços correntes, da Agropecuária (Vab_agr); renda per capita (renda_percapita); população total (pop_total); população rural (pop_rural) e área (área).

Segue abaixo a representação do modelo, referente a distribuição de estabelecimentos:

$E s t a b=\alpha+\beta_{1} v a b \_a g r+\beta_{2} v a b \_i n d+\beta_{2} r e n d a \_p e r c a p i t a+\beta_{4} p o p \_t o t a l+\beta_{5}$ pop_rural $+\beta_{6}$ área

A base de dados foi gerada através IBGE, do qual foram extraídas informações referentes ao setor agrícola como estabelecimento, VAB_ind e VAB_agr. Ainda no IBGE foram extraídos os dados das variáveis renda per capita; população total e rural; e por fim a área através da Produção Agrícola Municipal (PAM).

Para confirmar os resultados obtidos, serão acrescentadas cinco variáveis binárias, dummies, que assumem valor 0 ou $1^{5}$, com objetivo de classificar os municípios entre central e periférico (Tabela 1). A classificação foi realizada através do número de habitantes e da localização em região metropolitana e região periférica.

\section{Características}

\begin{tabular}{lccl}
\hline Dummies & Sigla & Habitantes & \multicolumn{1}{c}{ Localização } \\
\hline Cidade Grande Central & CGC & $>500.000$ & Região metropolitana \\
Cidade Média Central & CMC & $>10.000 \mathrm{e}<500.000$ & Região metropolitana \\
Cidade Pequena Central & CPC & $<10.000$ & Região metropolitana \\
Cidade Média Periférica & CMP & $>10.000 \mathrm{e}<500.000$ & Região periférica \\
Cidade Pequena Periférica & CPP & $<10.000$ & Região periférica \\
\hline
\end{tabular}

\footnotetext{
${ }^{4}$ Abate de reses, exceto suínos; Abate de suínos, aves e outros pequenos animais; Fabricação de produtos de carne; Preservação do pescado e fabricação de produtos do pescado; Fabricação de conservas de frutas; Fabricação de conservas de legumes e outros vegetais; Fabricação de sucos de frutas, hortaliças e legumes; Fabricação de óleos vegetais em bruto, exceto óleo de milho; Fabricação de óleos vegetais refinados, exceto óleo de milho; Fabricação de margarina e outras gorduras vegetais e de óleos não-comestíveis de animais; Preparação do leite; Fabricação de laticínios; Fabricação de sorvetes e outros gelados comestíveis; Beneficiamento de arroz e fabricação de produtos do arroz; Moagem de trigo e fabricação de derivados; Fabricação de farinha de mandioca e derivados; Fabricação de farinha de milho e derivados, exceto óleos de milho; Fabricação de amidos e féculas de vegetais e de óleos de milho; Fabricação de alimentos para animais; Moagem e fabricação de produtos de origem vegetal não especificados anteriormente; Fabricação de açúcar em bruto; Fabricação de açúcar refinado; Torrefação e moagem de café; Fabricação de produtos à base de café; Fabricação de produtos de panificação; Fabricação de biscoitos e bolachas; Fabricação de produtos derivados do cacau, de chocolates e confeitos; Fabricação de massas alimentícias; Fabricação de especiarias, molhos, temperos e condimentos; Fabricação de alimentos e pratos prontos; e Fabricação de produtos alimentícios não especificados anteriormente.

${ }^{5}$ Exemplo: CGC: 0: evento não ocorre; 1: município com mais de 500.000 habitantes e localizada em uma região metropolitana. CPP: 0: evento não ocorre; 1 município com menos de 10.000 habitantes e localizado em uma região periférica.
} 
Tabela 1: Classificação das dummies.

Fonte: Elaborada pelos autores.

O estudo utilizou os três estados da região do sul país, ou seja, Rio Grande do Sul, Santa Catarina e Paraná. A metodologia foi realizada através do software GeoDa e GeoDaSpace, para o ano de 2010. A matriz de peso que forneceu o maior I de Moran foi a matriz Rock.

\section{ANÁLISE DOS RESULTADOS}

A análise dos resultados está dividida em duas subseções, onde inicialmente são apresentados os resultados da análise exploratória de dados espaciais decorrente dos procedimentos metodológicos supracitados. Na segunda subseção, são apresentados os resultados derivados da modelagem econométrica espacial.

\section{Análise Exploratória de Dados Espaciais (AEDE)}

\section{I de Moran Global}

O primeiro passo da análise é verificar a presença de autocorrelação espacial entre os agentes através do índice I de Moran. Conforme Pimentel, Almeida e Sabbadini (2005), os valores que excedem o I de Moran calculado indicam que há autocorrelação espacial positiva, enquanto que valores abaixo do esperado indicam a presença de autocorrelação negativa. A Tabela 2 abaixo apresenta o coeficiente de I de Moran para o número de estabelecimentos das agroindústrias nos municípios da região sul do Brasil.

\begin{tabular}{c|c|c}
\hline Ano & I de Moran & Probabilidade \\
\hline 2010 & 0.1486 & 0.00000 \\
\hline
\end{tabular}

Tabela 2: Coeficiente de I de Moran para estabelecimentos de agroindústrias nos municípios da região sul do Brasil - 2010.

Fonte: Elaborada pelos autores.

O resultado mostra que o I de Moran é positivo (0.1486), o que indica autocorrelação espacial global positiva no período, ou seja, os agentes interagem entre si. Este resultado caracteriza os municípios com maior concentração de estabelecimentos agroindustriais na vizinhança de outros municípios com as mesmas peculiaridades. 
Outrossim, de modo complementar à análise do I de Moran tem-se o diagrama de dispersão de Moran. O diagrama revela os padrões locais de associação espacial, alocando as observações em quadrantes de acordo com a distribuição de estabelecimentos (VIDIGAL et al.; 2009). Uma vez que o I de Moran indica apenas uma tendência geral de agrupamento dos dados, o diagrama revela padrões locais de associação espacial (PEROBELLI et al.; 2007).

A vantagem do diagrama de dispersão de Moran é poder classificar as microrregiões de acordo com quatro diferentes regimes espaciais. Como observado na metodologia, eles podem ser: baixo-alto (BA); alto-alto (AA); alto-baixo (AB) e baixo-baixo (BB). A Figura 1 apresenta o diagrama para estabelecimentos agroindustriais, onde o eixo horizontal corresponde a variável em análise, e, o eixo vertical, a defasagem espacial (lag) da variável de interesse para o mesmo período. Como resultado tem-se o I Moran $(0,1399)$ positivo, presente principalmente no primeiro quadrante (AA).

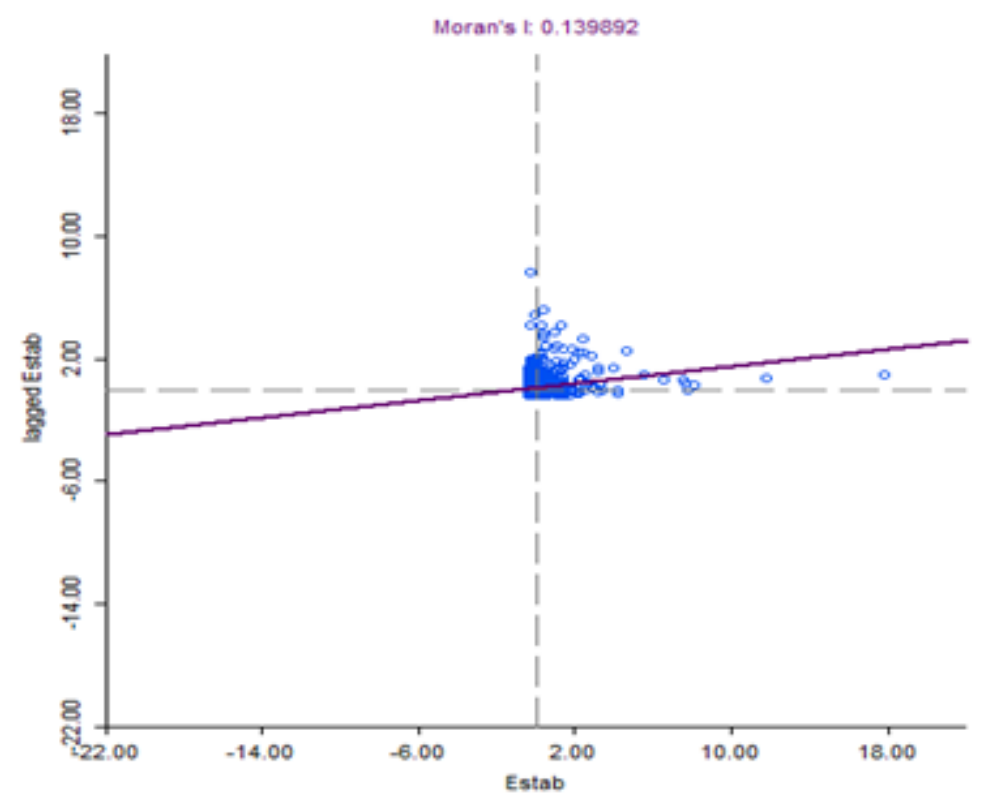

Figura 1: Diagrama de Dispersão de Moran para a variável endógena. Fonte: Elaborada pelos autores.

I de Moran Local

O próximo passo da análise consiste em examinar o Índice de Moran Local, com a função de capturar padrões locais de associação linear, realizando uma decomposição do indicador global de autocorrelação na contribuição local de cada observação em quatro categorias, cada qual correspondendo individualmente a um quadrante no diagrama de dispersão de Moran (ALMEIDA, 2004). De forma mais evidente, temos o mapa de clusters, 
que combina informações do mapa de dispersão de Moran e do mapa de significância das medidas de associação local.

Os indicadores locais de associação espacial (LISA) para o número de estabelecimentos agroindustriais nos municípios brasileiros da região sul para o ano de 2010 estão presentes no mapa de clusters (Figura 3). São identificados os seguintes padrões locais de clusters: Alto-Alto (64); Baixo-Baixo (36); Baixo-Alto (44) e Alto-Baixo (19), enquanto que os demais municípios não apresentam vizinhos com significância.
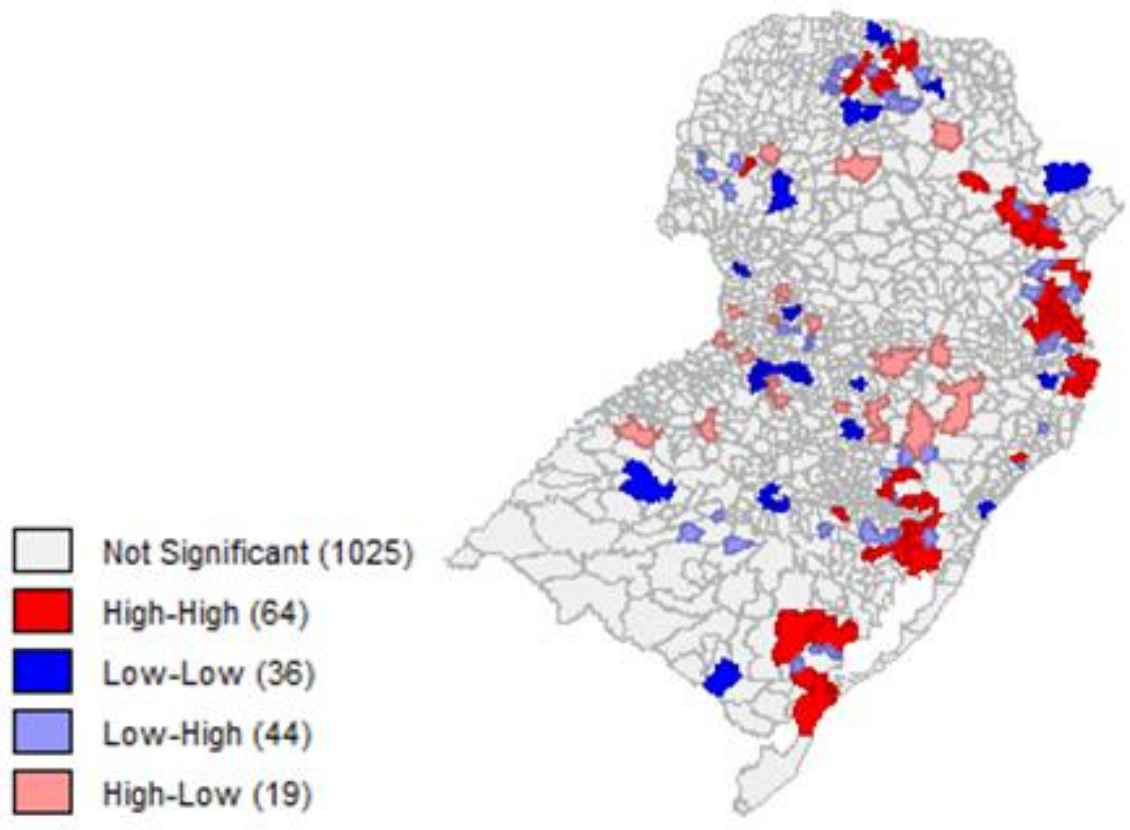

Figura 3: Mapa de cluster para estabelecimentos.

Fonte: Elaborada pelos autores.

No Mapa de cluster para estabelecimentos (Figura 3), o padrão (AA) prevalece na região sul do Brasil. No estado do Rio Grande do $\mathrm{Sul}^{6}$ é possível observar o padrão AA na região metropolitana de Porto Alegre, serra e região sul do estado. Em Santa Catarina ${ }^{7}$ na região da grande Florianópolis, Vale do Itajaí e norte catarinense; por fim no Paraná ${ }^{8}$, verifica-se o mesmo padrão para a região metropolitana de Curitiba, centro oriental paranaense e o norte central.

\footnotetext{
${ }^{6}$ Alvorada; Cachoeirinha; Campo Bom; Canela; Canguçu; Canoas; Capão do Leão; Dois Irmãos; Eldorado do Sul; Farroupilha; Gramado; Gravataí; Nova Petrópolis; Novo Hamburgo; Porto Alegre; Rio Grande; Rolante; São Lourenço do Sul; São Marcos; Sapucaia do Sul; Viamão; Taquara.

7 Balneário Camboriú; Biguaçu; Blumenau; Camboriú; Florianópolis; Forquilhinha; Garuva; Gaspar; Guaramirim; Ilhota; Indaial Itapema; Itajaí; Jaraguá do Sul; Luiz Alves; Massaranduba; Navegantes; Palhoça; Pomerode; Rodeio; São Francisco do Sul; São José; Santo Amaro da Imperatriz; Schroeder.

8 Almirante; Apucarana; Arapongas; Araucária; Astorga; Cafelândia; Cambé; Campo Largo; Carambeí; Colombo; Curitiba; Fazenda Rio Grande; Ibiporã; Marialva; São José dos Pinhais; Sertanópolis; Tamandaré.
} 
A Figura 3 revela que a concentração dos estabelecimentos agroindustriais ocorre em locais que possuem alta concentração populacional, ou seja, há uma aglomeração de agroindústrias em torno de cidades centrais. Isso implica em uma localização próxima do mercado consumidor e de rotas de transporte e longe da fonte de matérias-primas. De acordo com Belik e Vian (2005), as principais cadeias produtivas do agronegócio tem sua produção assimilada pelo consumo interno, sendo que a estabilidade do mercado interno contribui para o crescimento das exportações.

Para corroborar com estes resultados, foi realizado o mapa de clusters para as cinco dummies de localização, a fim de comprovar em que região específica ocorre as aglomerações. Por meio da Figura 4, observa-se que ocorre aglomeração de agroindústrias em torno de cidades centrais. Os clusters de padrão $\mathrm{AA}^{9}$ localizam-se nas proximidades (ou vizinhança) das cidades centrais.

De acordo com Elias e Pequeno (2007), o agronegócio atualmente está fortemente integrado à economia urbana e promovendo novas relações entre o campo e a cidade. Ainda segundo os autores, as cidades localizadas nas proximidades a áreas de realização do agronegócio são responsáveis pelo fornecimento de insumos de produção, como mão-de-obra, de recursos financeiros, assistência técnica, entre outros, o que promove as redefinições e fragmentações regionais.

No estado do Rio Grande do Sul as agroindústrias estão localizadas em torno da região metropolitana de Porto Alegre. No estado de Santa Catarina na região da grande Florianópolis, Vale do Itajaí e norte catarinense; enquanto no Paraná, na região metropolitana de Curitiba, centro oriental paranaense e o norte central. Todas as cidades de padrão AA foram classificadas como média central, ou seja, são municípios que apresentam uma população entre 10.000 e 500.000 habitantes e estão localizadas em regiões metropolitanas.

\footnotetext{
${ }^{9}$ Rio Grande do Sul: Alvorada; Arroio dos Ratos; Cachoeirinha; Canoas; Campo Bom; Capela de Santana; Charqueadas; Dois Irmãos; Eldorado do Sul; Estância Velha; Esteio; Gravataí; Igrejinha; Ivoti; Montenegro; Nova Santa Rita; Nova Hartz; Novo Hamburgo; Parobé; Portão; Rolante; Santo Antônio da Patrulha; São Jerônimo; São Leopoldo; Sapiranga; Sapucaia do Sul; Taquara; Triunfo; Viamão. Santa Catarina: Araquari; Biguaçu Campo Alegre; Corupá; Florianópolis; Garuva; Guaramirim; Itainópolis; Itapoá; Jaraguá do Sul; Mafra; Massaranduba; Palhoça; Rio Negrinho; Schroeder; São Bento do Sul; São Francisco do Sul; São José; Tijucas do Sul. Paraná: Almirante Tamandaré; Alvorada do Sul; Arapongas; Assaí; Balsa Nova; Bela Vista do Paraíso; Bocaiúva do Sul; Cambé; Campina Grande do Sul; Campo Largo; Campo Magro; Centenário do Sul; Colombo; Contenda; Fazenda Rio Grande; Florestópolis; Iporã; Itaperuçu; Jaguapitã; Jataizinho; Lapa; Mandirituba; Pinhais; Primeiro de Maio; Porecatu; Quitandinha; Quatro Barras; Rio Branco do Sul; Rolândia; São José dos Sertanópolis; Tijucas do Sul.
} 


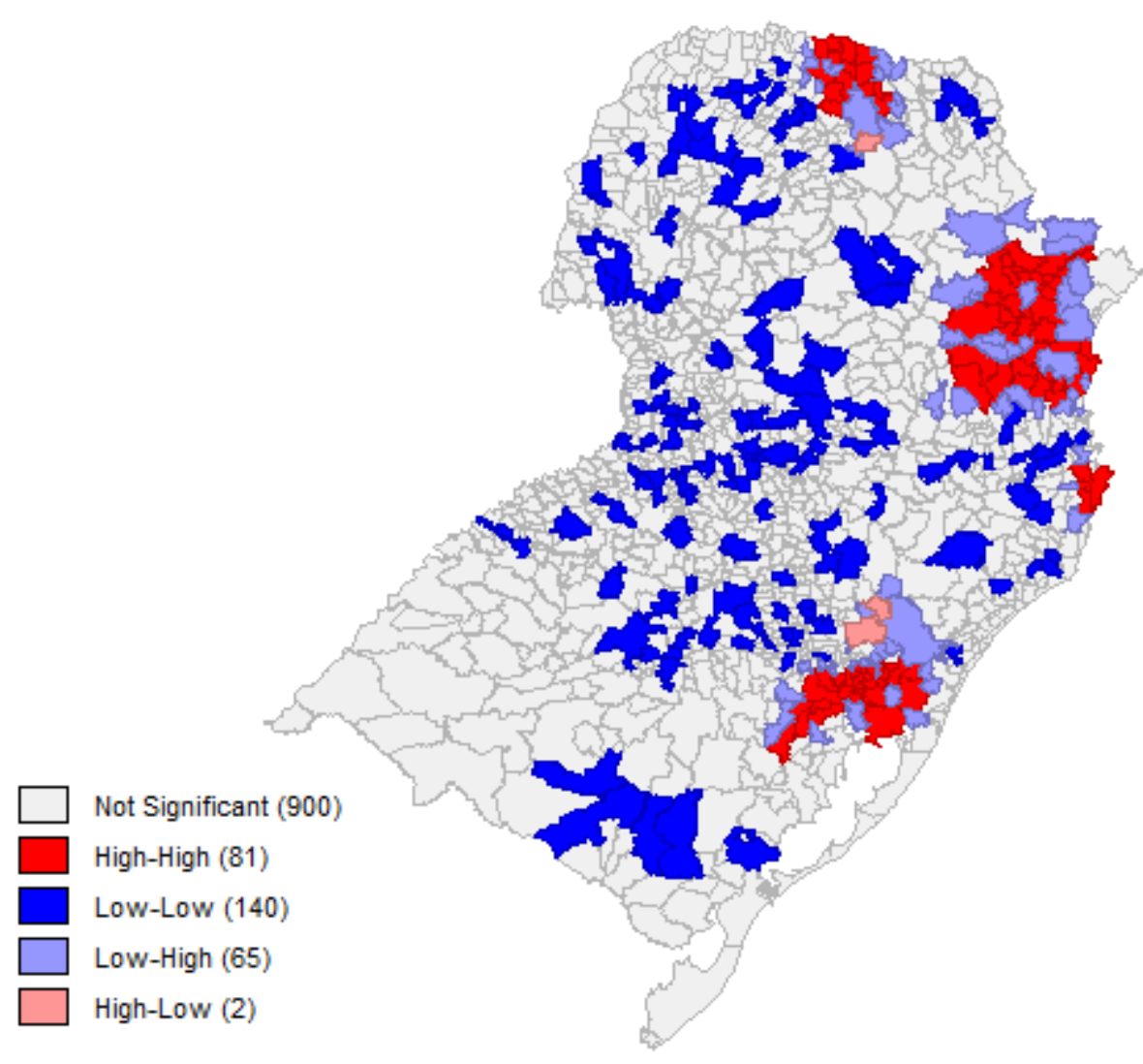

Figura 4: Mapa de cluster Cidade Média Central

Fonte: Elaborada pelos autores

\section{Econometria Espacial}

Com a questão espacial, torna-se primordial explicar as relações entre as variáveis, sendo que o uso do modelo clássico de regressão linear é considerado inadequado, uma vez que devem ser estimados modelos que considerem a dependência espacial (LINS et al., 2014). Na Tabela 3, observa-se o Modelo MQO em que todas as variáveis apresentam sinal positivo, com exceção da área que não é significativa no modelo.

Por meio do diagnóstico ${ }^{10}$ de dependência espacial é possível observar que ocorre dependência espacial no modelo, o qual indicou a utilização do modelo SAR e SEM ${ }^{11}$. No modelo espacial (Tabela 3), observa-se que todas as variáveis mostraram-se significativas, exceto área ${ }^{12}$, que não é significativa para explicar a disposição de estabelecimentos

\footnotetext{
${ }^{10} \mathrm{O}$ teste de Moran's I apresentou ser igual a 0.1486 (com significância de 0.0000 ). O modelo em MQO não apresentou multicolinearidade, porém apresentou heretocedasticidade, no qual foi utilizado o programa GeoDa Space para resolver o problema.

${ }^{11}$ Com o programa Geoda Space é possível utilizar os dois modelos juntos.

12 Testou-se o modelo sem área, porém não houve mudanças significativas.
} 
agroindustriais. O modelo mostra uma relação positiva entre o número de estabelecimentos e o Valor Adicionado Bruto agropecuário e o VAB industrial.

\begin{tabular}{|c|c|c|c|c|c|c|}
\hline \multirow[b]{2}{*}{ Variável } & \multicolumn{3}{|c|}{ MQO } & \multicolumn{3}{|c|}{$\begin{array}{l}\text { Modelo } \\
\text { Espacial }\end{array}$} \\
\hline & Coeficiente & Desvio Padrão & Prob & $\begin{array}{c}\text { Coeficient } \\
\text { e }\end{array}$ & Desvio Padrão & Prob \\
\hline & & & & $\begin{array}{c}- \\
10.256233\end{array}$ & & 0.00000 \\
\hline Constante & -11.8322 & 0.988535 & 0.00000 & $\begin{array}{c}1 \\
0.0000041\end{array}$ & 1.0957646 & $\begin{array}{c}0 \\
0.00067\end{array}$ \\
\hline Vab Agrícola & $3.24971 \mathrm{e}-005$ & $1.09159 \mathrm{e}-005$ & 0.00297 & 0 & 0.0000121 & $\begin{array}{c}9 \\
0.00686\end{array}$ \\
\hline Vab Industrial & $1.51814 \mathrm{e}-006$ & 7.16267e-007 & 0.03425 & 0.0000018 & 0.0000007 & $\begin{array}{c}5 \\
0.00000\end{array}$ \\
\hline Renda per capita & 0.018199 & 0.0013058 & 0.00000 & 0.0163542 & 0.0014553 & $\begin{array}{c}0 \\
0.00000\end{array}$ \\
\hline Pop total & 0.000226904 & $5.17387 \mathrm{e}-006$ & 0.00000 & 0.0002292 & 0.0000049 & $\begin{array}{c}0 \\
0.00000\end{array}$ \\
\hline Pop rural & $\begin{array}{c}0.000674685 \\
-1.05699 \mathrm{e}-\end{array}$ & $8.50038 \mathrm{e}-005$ & 0.00000 & 0.0007178 & 0.0000891 & $\begin{array}{c}0 \\
0.37975\end{array}$ \\
\hline Área & $005^{*}$ & $1.6909 \mathrm{e}-005$ & 0.53203 & $-0.000017^{*}$ & 0.0000196 & $\begin{array}{c}2 \\
0.00025\end{array}$ \\
\hline W_Estab & & & & -0.0781940 & 0.0213674 & $\begin{array}{c}2 \\
0.00000\end{array}$ \\
\hline Lambda & & & & 0.3492089 & 0.0317201 & 0 \\
\hline $\mathbf{R}^{2}$ & 0.8609 & & & 0.8624 & & \\
\hline
\end{tabular}

Tabela 3: Resultados para a estimação do Modelo MQO e Modelo Espacial. Fonte: Elaborada pelos autores.

Nota: * Não significativo.

O mesmo ocorre com a população rural e total que apresentam associação positiva com o número de estabelecimentos. Enquanto, o aumento de 10.000 habitantes na população total promove uma variação positiva de dois estabelecimentos. $\mathrm{O}$ aumento na mesma proporção na população rural resulta em um ganho de sete estabelecimentos.

A renda per capita tem associação positiva com o número de estabelecimentos, donde um aumento de $\mathrm{R} \$ 10.000,00$ na renda per capita promove um aumento de, aproximadamente, 164 estabelecimentos nas agroindústrias.

\section{CONSIDERAÇÕES FINAIS}

O artigo se propôs a verificar os padrões geográficos das indústrias do agronegócio, sendo realizada a análise por meio do número de estabelecimentos dispostos nos municípios da região sul, a fim de apurar se ocorre o fenômeno de concentração/aglomeração agroindustrial em torno das cidades centrais. 
Como base metodológica foi utilizada a AEDE e econometria espacial, sendo que a aplicação da análise exploratória permitiu verificar a presença de correlação espacial para o ano de 2010, no qual o resultado do teste de $I$ de Moran Global apresentou-se positivo e estatisticamente significativo, indicando dependência espacial positiva em relação às variáveis em estudo. Por meio do indicador LISA foram identificados os seguintes padrões locais de clusters: Alto-Alto (64); Baixo-Baixo (36); Baixo-Alto (44) e Alto-Baixo (19), enquanto que os demais municípios não apresentam vizinhos com significância. Ou seja, 14\% de vizinhos que apresentam clusters significativos.

Prevaleceu a formação de clusters do tipo alto-alto (AA), localizados em regiões importantes dos estados em estudo. No Rio Grande do Sul, foi possível observar clusters na região metropolitana de Porto Alegre, serra e a região sul do estado. As três regiões são importantes, uma vez que a região metropolitana e serra apresentam vantagens logísticas, com fácil acesso para todas as demais regiões e constituem-se como fornecedoras de insumos, como mão-de-obra qualificada. A região sul do estado apresenta rota de escoamento da produção, principalmente devido a localização próxima ao porto.

O mesmo padrão ocorre com o estado de Santa Catarina, que apresenta clusters altoalto na região da Grande Florianópolis, Vale do Itajaí e norte catarinense. No estado do Paraná a concentração ocorre na região metropolitana de Curitiba, centro oriental paranaense e norte central paranaense.

Para confirmar as regiões que ocorrem aglomeração de agroindústrias foram utilizadas cinco dummies, confirmando em algumas regiões que ocorre aglomeração de agroindústrias em torno de cidades centrais. A dummy que se mostrou mais próxima foi cidade média central, sendo que no Rio Grande do Sul estão localizadas em torno da região metropolitana de Porto Alegre, porém não se confirmou clusters AA na região da serra e no sul do estado. No estado de Santa Catarina na região da grande Florianópolis, Vale do Itajaí e norte catarinense; enquanto no Paraná, na região metropolitana de Curitiba, centro oriental paranaense e o norte central.

Após a aplicação da AEDE com a identificação dos clusters, procedeu-se às estimação do modelo econométrico. O modelo de MQO clássico indicou que os modelos SAR e SEM seriam os mais adequados para análise. O modelo indicou sinal positivo e significativo para as variáveis $\mathrm{VAB}$ agropecuário e da indústria; renda per capita; população total e rural; enquanto a área apresentou sinal negativo, porém não insignificante no modelo. 
Os resultados indicam que as agroindústrias estão se concentrando nos principais centros de cada estado em estudo, ou seja, as empresas estão próximas de locais com maior população, o mercado consumidor, e locais fornecedores de insumos de produção. Desta constatação verifica-se certa dissonância com teoria Weberiana, que pressupõem a concentração industrial próxima das fontes de matéria-prima, o que minimizaria os custos de transporte.

Para trabalhos futuros é possível incluir variáveis que façam referências aos custos de transporte e demais fatores que possam captar as principais motivações na instalação de agroindústrias em determinadas regiões. Também podem ser analisadas cadeias específicas de produtos, uma vez que as peculiaridades dos produtos agroindustriais delimitam o uso de abordagens convencionais.

\section{REFERÊNCIAS}

ALMEIDA, E. S. Curso de Econometria Espacial Aplicada. Piracicaba, 2004.

ANSELIN, L. SpaceStat Tutorial: A Workbook for Using SpaceStat in the Analysis of Spatial Data. 1992. Disponível em: http://www.spacestat.com.docs/spacestat_tutorial.pdf; Acesso em: 12 jul. 2017.

ANSELIN, L. Local indicators of spatial association - LISA. Geographical Analysis, v. 27, n. 2, p. 93-115, 1995.

AZZONI, C. R. Teoria da localização: análise crítica a partir das evidências empíricas no Estado de São Paulo. 1982. 287 p. Tese (Doutorado), Universidade de São Paulo, São Paulo.

BELIK, W.; VIAN, C. E. de F. Agricultura, Comércio Internacional e Consumo de Alimentos no Brasil. In: Congresso da Sociedade Brasileira de Economia e Sociologia Rural, 43, Ribeirão Preto. Anais... Ribeirão Preto: Sociedade Brasileira de Economia e Sociologia Rural (SOBER), 2005.

BRASIL. Ministério da Agricultura, Pecuária e Abastecimento. Estatísticas de Comércio Exterior do Agronegócio Brasileiro. Disponível em: <http://indicadores.agricultura.gov.br/index.htm>. Acesso em 12 jul. 2017.

CÂMARA, G.; MONTEIRO, A. G.; FUCKS, S. D.; CARVALHO, M. S. (2000) Análise Espacial e Geoprocessamento. In: Análise Espacial de Dados Geográficos, eds. FUCKS, S. D.; CARVALHO, M. S.; CÂMARA, G. Disponível em: < http://www.dpi.inpe.br/gilberto/livro/analise/ >. Acesso em: 20 maio. 2016.

CANUTO, A. Agronegócio: a modernização conservadora que gera exclusão pela produtividade. Revista Nera, v. 7, n. 5, p. 1-12, 2004.

CARMO, E. A. do; SOARES, J. B.; LOPES, M. A. Estudo da localização de abatedouros e centros de distribuição de agroindústrias de frangos de corte no Distrito Federal. Boletim de Indústria Animal, v. 68, n. 2, p. 101-112, 2011.

CONFEDERAÇÃO DA AGRICULTURA E PECUÁRIA DO BRASIL. PIB do Agronegócio Brasileiro. Disponível em: 〈http://cepea.esalq.usp.br/pib/>. Acesso em: 03 jul. 2017.

ELIAS, D.; PEQUENO, R.. Desigualdades socioespaciais nas cidades do agronegócio. Revista Brasileira de Estudos Urbanos e Regionais, v. 9, n. 1, p; 25-39, 2007. 
GASQUES, J. S.; BASTOS, E.T.; BACCHI, M. P. R.; CONCEIÇÃO, J. C. P. R. Produtividade e Fontes de Crescimento da Agricultura Brasileira. Revista de Política Agrícola, v. 13, p. 73-90, 2004.

GUIMARÃES, M. F. Desenvolvimento regional, efeito de localização e clusters agroindustriais no Brasil. Revista de Política Agrícola, v. 15, n. 2, p. 56-62, 2006.

HADDAD, P. R. A concepção de desenvolvimento regional. In: HADDAD, P. R. et al. (Org.). A competitividade do agronegócio e o desenvolvimento regional no Brasil: estudos de clusters. Brasília: CNPq: Embrapa, 1999.

de análise espacial. BNB/ETENE: Fortaleza, 1989.

INSTITUO BRASILEIRO DE GEOGRAFIA E ESTATÍSTICA. Fonte de dados. Disponível em: 〈http://www.ibge.gov.br/home>. Acesso em: 20 abr. 2016.

KUSBICK, F. A.; TARTAS, R. L. Fatores que influenciaram a implantação e desenvolvimento da agroindústria sob a ótica de Alfred Weber: O caso do oeste catarinense. Revista Cadernos de Economia, v. 19, n. 35, p. 47-64, 2016.

LE GALLO, J.; ERTHUR, C. Exploratory spatial data analysis of the distribution of regional per capita.GDP in Europe, 1980-1995.Papers in Regional Science v 82(2) p 175- 201. 2003.

LINS, J. G. M. G.; LOURES, A. R.; LOMBARDI FILHO, S. C.; SILVA, M. V. B. Análise espacial da evolução do índice de desenvolvimento humano nos municípios da região nordeste.In: X Encontro de Economia Baiana, Anais, 2014.

LOPES, R. L.; CAIXETA FILHO, J. V. Suinocultura no Estado de Goiás: aplicação de um modelo de localização. Pesquisa Operacional, v. 20, n. 2, p. 213-232, 2000.

LÖSCH, A. The economics of location. New Haven: Yale University Press, 1954.

OSTROSKI, D. A.; MEDEIROS, N. H. Cluster agroindustrial: fortalecimento e competitividade para a cadeia suinícola do município de Toledo. In: SOBER - Sociedade Brasileira de Economia e Sociologia Rural. Anais do XLII Congresso Brasileiro de Economia e Sociologia Rural. Cuiabá: SOBER, 2004.

PEROBELli, F. S.; ALMEIDA, E. S. de; ALVIM, M. I. da S.; FERREIRA, P G. C. Produtividade do setor agrícola brasileiro (1991-2003): uma análise espacial. Nova economia, v. 17, n.1, p.65-91, 2007.

PIMENTEL, E. A.; ALMEIDA, L. O.; SABBADINI, R. Comportamento Recente das Exportações Agrícolas no Brasil: Uma Análise Espacial no Âmbito dos Estados. In: Congresso da Sociedade Brasileira de Economia e Sociologia Rural, 43, Ribeirão Preto. Anais... Ribeirão Preto: Sociedade Brasileira de Economia e Sociologia Rural (SOBER), 2005.

PRADO, F. A.; BERVEGLIERI, A.; TACHIBANA, V. M.; IMAI, N. N. Aplicação e análise de modelos de regressão clássica e espacial para os distritos da cidade de São Paulo. In: III Simpósio Brasileiro de Ciências Geodésicas e Tecnologias da Geo informação, Recife. Anais, 2010.

RICHARDSON, H. W. Economia regional. Rio de Janeiro: Zahar, 1981.

ROCHA, C. B.; PARRÉ, J. L. Estudo da distribuição espacial do setor agropecuário do Rio Grande do Sul. Análise Econômica, Porto Alegre, v. 27, n. 52, p. 139-160, 2009.

SOUZA, R. M.; PEROBELLI, F. S. Diagnóstico espacial da concentração produtiva do café no Brasil, no período de 1991 a 2003. Revista de Economia e Agronegócio, vol. 5, n. 3, p 353-378, 2007.

TOREZANI, T. A.; ANDREOTTI, A. de Q. A.; CAMPOS, A. C. de. Estudo da dinâmica agrícola na microrregião de Paranavaí-PR: um aporte da teoria clássica da localização. Revista Paranaense de Desenvolvimento-RPD, v. 34, n. 124, p. 201-224, 2013.VIDIGAL, V. G.; GOMES, M. F. M.; ROCHA-VIDIGAL, C. B.; SILVEIRA, G. F. Análise espacial da 
produtividade da cana-de-açúcar em Minas Gerais, 1990 a 2007. Revista de Economia e Administração, v.10, n.2, p. 241-261, 2011.

WEBER, A. Theory of the Location of Industries. University of Chicago Press, 1929.

Recebido em 16 de novembro de 2017.

Aceito em 17 de abril de 2018. 